\title{
Comunicación
}

\section{Uropatía obstructiva en canino: reporte de caso clínico}

\author{
Obstructive uropathy in canine: clinical case report
}

\author{
Juan Carlos Pinilla León ${ }^{1,3}$, Natalia Da Silva ${ }^{2}$
}

\section{Resumen}

\begin{abstract}
El presente estudio muestra el caso de un canino macho de 7 años que ingresó de emergencia a la consulta de la clínica veterinaria «Vet Center», Bucaramanga, Colombia, por un presunto cuadro de envenenamiento. El paciente mostraba signos de depresión, disuria, estranguria, hematuria y vómito. La valoración hematológica y urianálisis revelaron un cuadro de deshidratación e infección urinaria. La química sanguínea demostró insuficiencia y azotemia posrenal, y el examen ecográfico mostró la presencia de un urolito y repleción de la vejiga urinaria, confirmando el diagnóstico de uropatía obstructiva. Para la estabilización del paciente se colocó sonda urinaria Nelaton para vaciado de la vejiga e hidropropulsión; así como aplicación de soluciones endovenosas de mantenimiento con $\mathrm{NaCl}$ al $0.9 \%$, antibióticos y antiinflamatorios. Finalmente, se realizó cistotomía para remoción del urolito, además de una uretrostomía debido a la recurrencia de la obstrucción.
\end{abstract}

Palabras clave: caninos; uremia; uropatía

\section{AbStract}

The present study shows the case of a 7-year-old male canine who was admitted to the veterinary clinic «Vet Center», Bucaramanga, Colombia, for an alleged poisoning picture. The patient showed signs of depression, dysuria, strangury, haematuria and vomiting. Haematological assessment and urinalysis revealed a picture of dehydration and urinary infection. Blood chemistry showed insufficiency and postrenal azotemia,

\footnotetext{
${ }^{1}$ Universidad de Santander, Facultad de Ciencias Exactas, Naturales y Agropecuarias, Grupo de Investigación en Ciencias Agropecuarias-GICA, Bucaramanga, Colombia

${ }^{2}$ Universidad Rómulo Gallegos, Facultad de Agronomía, Departamento de Parasitología, San Juan de Los Morros, Venezuela

${ }^{3}$ E-mail: jcpinilla@hotmail.com
}

Recibido: 14 de marzo de 2019

Aceptado para publicación: 12 de noviembre de 2019 
and the ultrasound examination showed the presence of a urolith and urinary bladder repletion, confirming the diagnosis of obstructive uropathy. For the stabilization of the patient, a Nelaton urinary catheter was placed to empty the bladder and hydro propulsion was done. Besides, maintenance intravenous solutions with $0.9 \% \mathrm{NaCl}$, antibiotics and anti-inflammatory were prescribed. Finally, cystotomy was performed to remove the urolith, in addition to a urethrostomy due to the recurrence of the obstruction.

Key words: canines; uremia; uropathy

\section{INTRODUCCIÓN}

Las uropatías del tracto urinario inferior de los perros incluyen varias enfermedades que afectan la vejiga, la uretra y la próstata; siendo los signos clínicos más comunes en estas enfermedades la disuria, hematuria, estranguria y polaquiuria (Bartges, 2004). Dentro de estas enfermedades, la obstrucción uretral es una patología que ocurre con mayor frecuencia en los machos, generalmente asociada con enfermedades benignas o malignas. Las primeras pueden ser causadas por urolitiasis, disinergia refleja y uretritis granulomatosa (Moroff et al., 1991; DiazEspineira et al., 1998; Hostutler et al., 2004), además de neoplasias y traumatismos, los cuales generan estenosis uretral, que pueden llevar a la muerte del paciente por un cuadro de uremia posrenal. Bartges (2004) indica que las infecciones urinarias y la urolitiasis son las enfermedades más frecuentes en perros de los Estados Unidos; sin embargo, Inkelmann et al. (2012) reportaron mayor frecuencia de lesiones inflamatorias y uropatías obstructivas en perros del Brasil.

La uretrotomía es un procedimiento quirúrgico que consiste en realizar una incisión en el lumen uretral para extirpar o aliviar temporalmente una obstrucción. Sin embargo, cuando se requiere un desvío permanente del flujo de orina proximal a una uretra obstruida se realiza una uretrostomía para aliviar permanentemente la obstrucción uretral (Smeak, 2000). En el perro puede realizarse a nivel prescrotal, escrotal, perineal y subpúbica
(Bernarde y Viguier, 2004; Bleedom y Bjorling, 2012). En este procedimiento quirúrgico se crea un desvío permanente de la orina mediante la sutura de la mucosa del pene a la piel después de la exposición de la luz de la uretra. Las complicaciones asociadas con la uretrostomía incluyen formación de estenosis, hemorragia, dehiscencia, pérdida de orina, hernia perineal, incontinencia urinaria o fecal, prolapso rectal e infección del tracto urinario ascendente (Milgram, 2017).

\section{Caso Clínico}

Se describe el caso clínico de un canino, macho entero, raza Schnauzer de 7 años, $8.2 \mathrm{~kg}$ de peso corporal, que ingresó a la emergencia de la Clínica Veterinaria «Vet Center», en Bucaramanga, Colombia, por un presunto cuadro de envenenamiento. El propietario informó que su perro había vomitado varias veces durante la noche anterior, y que no come ni defeca desde hace dos días. Igualmente informó que orina con cierta dificultad y por gotas.

\section{Examen Clínico}

En la valoración física, el paciente se mostró deprimido, buena condición corporal, $6 \%$ de deshidratación, 2 segundos en tiempo de llenado capilar, mucosas oculares de color normal, temperatura corporal (TC): $39.9^{\circ} \mathrm{C}$, frecuencia respiratoria (FR): 72 movimientos respiratorios /min y frecuencia cardíaca (FC): 100 latidos/min. El paciente presenta- 
ba taquipnea, pulso arterial fuerte (arteria femoral). A la palpación, los linfonódulos superficiales se mostraron sin alteraciones patológicas; sin embargo, el paciente manifestaba abdomen defensivo y tenso, con gran repleción de la vejiga urinaria, lo que indicaba la emergencia del caso. Al examen clínico del sistema urinario se observó un paciente con disuria, hematuria y estranguria, confirmando uno de los motivos de la consulta.

Con base a la información obtenida en la historia médica y el examen físico, se estableció como diagnósticos diferenciales la urolitiasis, cistitis bacteriana y obstrucción uretral; por tanto, se solicitó un estudio ecográfico de abdomen craneal y caudal, además de exámenes de laboratorio, hematología completa, enzimas séricas para evaluar funcionabilidad renal y uroanálisis. En vista de tratarse de un caso de urgencias, se procedió a internar el paciente en la clínica veterinaria para su manejo clínico hospitalario

\section{Manejo Terapéutico de Sostén}

Inicialmente se colocó una sonda uretral Nelaton, calibre N. ${ }^{\circ} 5$, de $40 \mathrm{~cm}$ de longitud, para el vaciado de la vejiga urinaria; sin embargo, se tuvo que tranquilizar al paciente con $0.05 \mathrm{mg} / \mathrm{kg}$ PV $(0.6 \mathrm{ml})$ de acepromazina (Tranquilan 1\%) por vía i.m. y $7 \mathrm{mg} / \mathrm{kg}$ PV $(1.1 \mathrm{ml})$ de clorhidrato de ketamina (Ketamina $5 \%)$ por vía endovenosa. La entrada de la sonda por el canal uretral ofreció resistencia; realizándose hidropropulsión con solución de $\mathrm{NaCl}$ al $0.9 \%$. A través de la sonda uretral se realizaron lavados con solución salina $0.9 \%$ y metronidazol $(5 \mathrm{ml})$ a intervalos de 6 horas, de manera de permeabilizar la vía urinaria. A las 48 horas se retiró la sonda; sin embargo, nuevamente se obstruyó el canal uretral, por lo que se recurrió a la cirugía.

El paciente recibió fluidoterapia endovenosa de mantenimiento con $\mathrm{NaCl}$ al $0.9 \%$ (45 ml $/ \mathrm{kg}$ ), enrofloxacina por vía endovenosa (10 mg/kg, c/24 horas), metronidazol (50 mg/ $\mathrm{kg}$, diluidos en la solución de $\mathrm{NaCl}, \mathrm{c} / 12$ horas) y dipirina ( $0.4 \mathrm{ml}$, vía endovenosa, c/8 horas).
Cuadro 1. Resultados de la hematología completa en el paciente canino con uropatía obstructiva

\begin{tabular}{lcc}
\hline Parámetro & Resultado & Referencia $^{1}$ \\
\hline Glóbulos rojos & $7.5 \times 10^{6}$ & $5.4-10,3$ \\
Hematocrito (\%) & 50 & $37-45$ \\
Hemoglobina (mg\%) & 16.2 & $11.8-14.5$ \\
Leucocitos (x mm $\left.{ }^{3}\right)$ & 15,000 & $6,000-11,000$ \\
Fórmula diferencial & & \\
Neutrófilos (\%) & 85 & $60-70$ \\
Linfocitos (\%) & 12 & $12-30$ \\
Eosinófilos (\%) & 3 & $1-5$ \\
Monocitos (\%) & 0 & $0-2$ \\
Bandas (\%) & 0 & $0-2$ \\
Plaquetas (x mm $\left.{ }^{3}\right)$ & 313,000 & $150,000-$ \\
& & 500,000 \\
\hline
\end{tabular}

${ }^{1}$ Pedrozo et al. (2010)

\section{Estudio de Laboratorio}

Los resultados de la hematología (Cuadro 1) muestran un aumento de los valores de hematocrito (50\%) y hemoglobina (16.2 mg\%), lo que indica un cuadro de deshidratación. La serie de células blancas muestra una leucocitosis $\left(15000 \times \mathrm{mm}^{3}\right)$ con neutrofilia $(85 \%)$, lo que indica la presencia de un proceso infeccioso bacteriano de evolución aguda. Los valores de plaquetas se encontraron dentro de los parámetros normales.

En el Cuadro 2 se muestran los resultados de química sanguínea para valoración renal. Los valores de creatinina se encontraron dentro de los valores de referencia; sin embargo, el nitrógeno ureico sanguíneo (BUN) resultó aumentado $(51 \mathrm{mg} / \mathrm{dl})$, lo que indicaba un problema de insuficiencia y azotemia posrenal. Las altas concentraciones de urea en sangre ocasionaban en el paciente la presencia de vómito, depresión y anorexia.

La orina presentó color ámbar y alta turbidez. En el examen fisicoquímico (Cuadro 3 ) se determinó presencia de cetonas ( 8.8 $\mu \mathrm{mol} / \mathrm{l})$, provenientes del metabolismo de las 
Cuadro 2. Resultados de química sanguínea en el paciente canino con uropatía obstructiva

\begin{tabular}{lcc}
\hline Parámetro & Resultado & Referencia $^{1}$ \\
\hline Creatinina & 1.38 & $0.6-1.5$ \\
$\begin{array}{l}\text { Nitrógeno ureico } \\
\text { sanguíneo (BUN) }\end{array}$ & 51 & $8-29$ \\
\hline
\end{tabular}

${ }^{1}$ Pedrozo et al. (2010)

Cuadro 3. Resultados de la evaluación fisicoquímica de la orina en el paciente canino con uropatía obstructiva

\begin{tabular}{lcc}
\hline Parámetro & Resultado & Referencia $^{1}$ \\
\hline Cetonas, $\mu \mathrm{mol} / 1$ & 8.8 & $0.5-1$ \\
Nitritos & + & - \\
Urobilinógeno, $\mu \mathrm{mol} / 1$ & 33 & 3.2 \\
Bilirrubina, $\mu \mathrm{mol} / 1$ & 33 & $0-2$ \\
Glucosa, $\mathrm{mg} \%$ & 2.8 & $17-22$ \\
Proteína & +++ & - \\
Densidad específica & 1015 & $1010-1070$ \\
pH & 7 & $5-7.5$ \\
Sangre, cell/1 & 80 & $3-8$ \\
Ác ascórbico, $\mu \mathrm{mol} / 1$ & 1.4 & 0.6 \\
Microalbúmina, mg/l & 30 & $<10$ \\
Calcio, $\mu \mathrm{mol} / \mathrm{l}$ & 10 & $<1$ \\
Creatinina, $\mu \mathrm{mol} / 1$ & 26.4 & 17.7 \\
\hline
\end{tabular}

${ }^{1}$ Pedrozo et al. (2010)

Cuadro 4. Resultados del examen microscópico del sedimento urinario en el paciente canino con uropatía obstructiva

\begin{tabular}{lc}
\hline Parámetro & Resultado \\
\hline Bacterias & Abundantes \\
Leucocitos & $12-16 /$ campo \\
Cilindros de hematíes & $2-4$ \\
Hematíes & $14-20 /$ campo \\
Células renales & $0-2$ \\
Células epiteliales & $18-22 /$ campo \\
Cristales de estruvita & +++ \\
\hline
\end{tabular}

grasas, en vista que el paciente tenía dos días sin comer. Además, se encontró proteinuria $(+++)$, microalbuminuria $(30 \mathrm{mg} / \mathrm{l})$ y hematuria (80 cél/1), lo que indicaba daño renal (Cortadellas y Fernández del Palacio, 2012) $\mathrm{y}$ un proceso inflamatorio a nivel de vejiga urinaria. En el examen del sedimento se encontraron abundantes bacterias, leucocitos, hematíes y células epiteliales (Cuadro 4), lo que confirmaba la presencia del cuadro infeccioso. También se observaron cristales de estruvita que señalaba la presencia de sedimento urinario.

\section{Estudio Ecográfico}

La vejiga presenta paredes delgadas y gran volumen de contenido luminal anecoico. En el lumen se muestra una estructura pequeña y de aspecto hiperecoico con $0.41 \mathrm{~cm}$ de diámetro que sugiere la presencia de urolito (Figura 1A). Así mismo, se evidencia turbidez del contenido luminal de la vejiga urinaria, y engrosamiento leve de la pared del conducto urinario en la región del trígono vesical (Figura 1B). En la exploración del mesogastrio se identifica el riñón izquierdo con variación del patrón ecogénico y alteración de la relación corteza-médula, lo que sugiere pielonefritis. El riñón derecho se identifica bajo patrones normales de ecogenicidad $\mathrm{y}$ forma.

\section{Manejo Quirúrgico}

Inicialmente se realizó una cistotomía a través de una laparotomía paramedial para realizar la extracción del urolito. La gran repleción de la vejiga urinaria no permitió su salida por la herida quirúrgica, por lo que se realizó la cistocentesis para su vaciado y posterior abordaje quirúrgico (Figura 2A,B).

La vejiga urinaria se lavó con solución salina y metronidazol, y se colocó una sonda en sentido cráneo caudal para hacer el lavado uretral por hidropropulsión. A las 24 horas de la cirugía (cistotomía), el paciente nueva- 


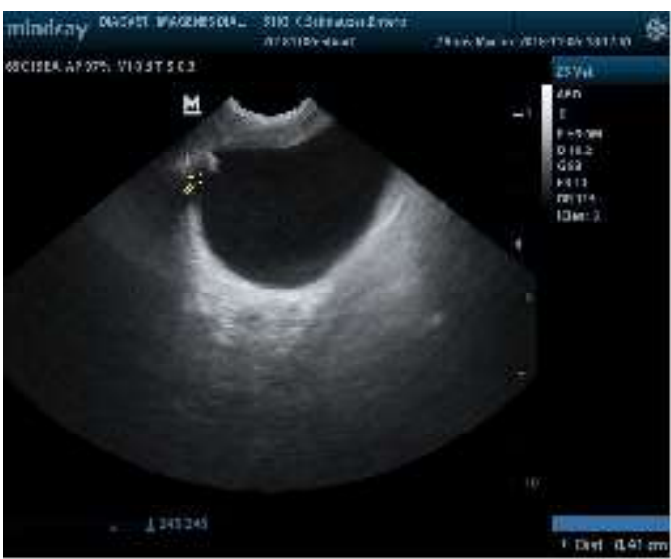

(A)

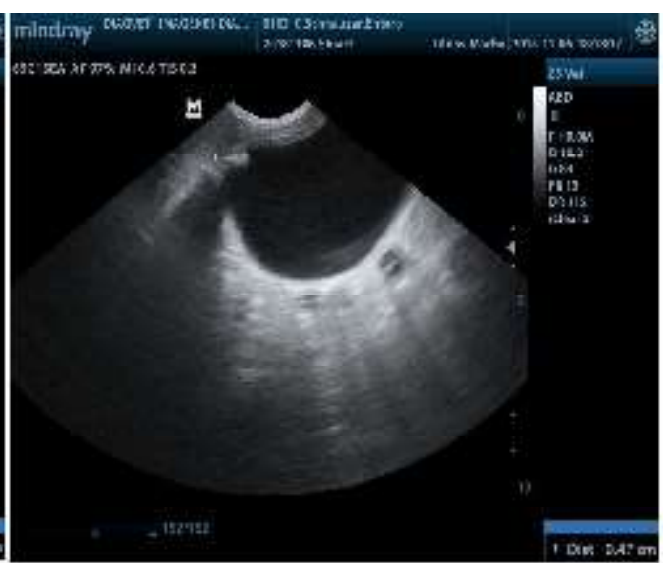

(B)

Figura 1. Imagen ecográfica del paciente perro macho Schnauzer de 7 años. (A) Presencia de urolito en vejiga (flecha). (B) Engrosamiento de la pared vesical

mente manifestó obstrucción uretral total. Se procedió a realizar una uretromía preescrotal con lavados en ambos sentidos (Figura 2C,D); sin embargo, hubo recurrencia de la obstrucción probablemente debido a la presencia de alguna placa de minerales que se haya formado con el tiempo, o por algún problema de disinergia del detrusor uretral (Bleedom y Bjorling, 2012). Finalmente se realizó uretrostomía preescrotal y castración del paciente (Figura E,F). Se presentó hemorragia las primeras 24 horas posteriores a la cirugía; sin embargo, se suministró $1 \mathrm{ml}$ de vitamina $\mathrm{K}$ vía subcutánea $(5 \mathrm{mg} / \mathrm{kg} \mathrm{PV})$ cada 12 horas por tres días. También se manejaron antibióticos y analgésicos durante una semana para evitar complicaciones.

Los signos de uremia y demás signos clínicos desaparecieron luego de la cirugía. El propietario ha seguido las indicaciones para evitar infecciones urinarias, además que bimensualmente llevaron el paciente a control médico. El paciente no tuvo recurrencia de obstrucción uretral, evolucionando satisfactoriamente. Se concluye que la urestrostomía preescrotal es una técnica quirúrgica que puede ser empleada en casos de obstrucción uretral permanente

\section{Agradecimientos}

Los autores agradecen al propietario del paciente por la colaboración prestada en la ejecución del presente trabajo clínico. También se agradece a la Universidad de Santander por el apoyo prestado en la redacción del presente artículo.

\section{Literatura Citada}

1. Bartges $J W .2004$. Diagnosis of urinary infections. Vet Clin North Am Small Anim Pract 34: 923-933. doi: 10.1016/ j.cvsm.2004.03.001

2. Bernarde A, Viguier E. 2004. Transpelvic urethrostomy in 11 cats using an ischial ostectomy. Vet Sur-g 33: 246252. doi: 10.1111/j.1532-950X.2004.04036.x

3. Bleedom JA, Bjorling DE. 2012. Urethra. In: Tobias KM, Johnston SA (eds). Veterinary surgery - Small ani-mal. St. Louis, USA: Elsevier. p 1993-2010.

4. Cortadellas OM, Fernández del Palacio J. 2012. Diagnóstico y tratamiento de la enfermedad renal crónica (ERC) 

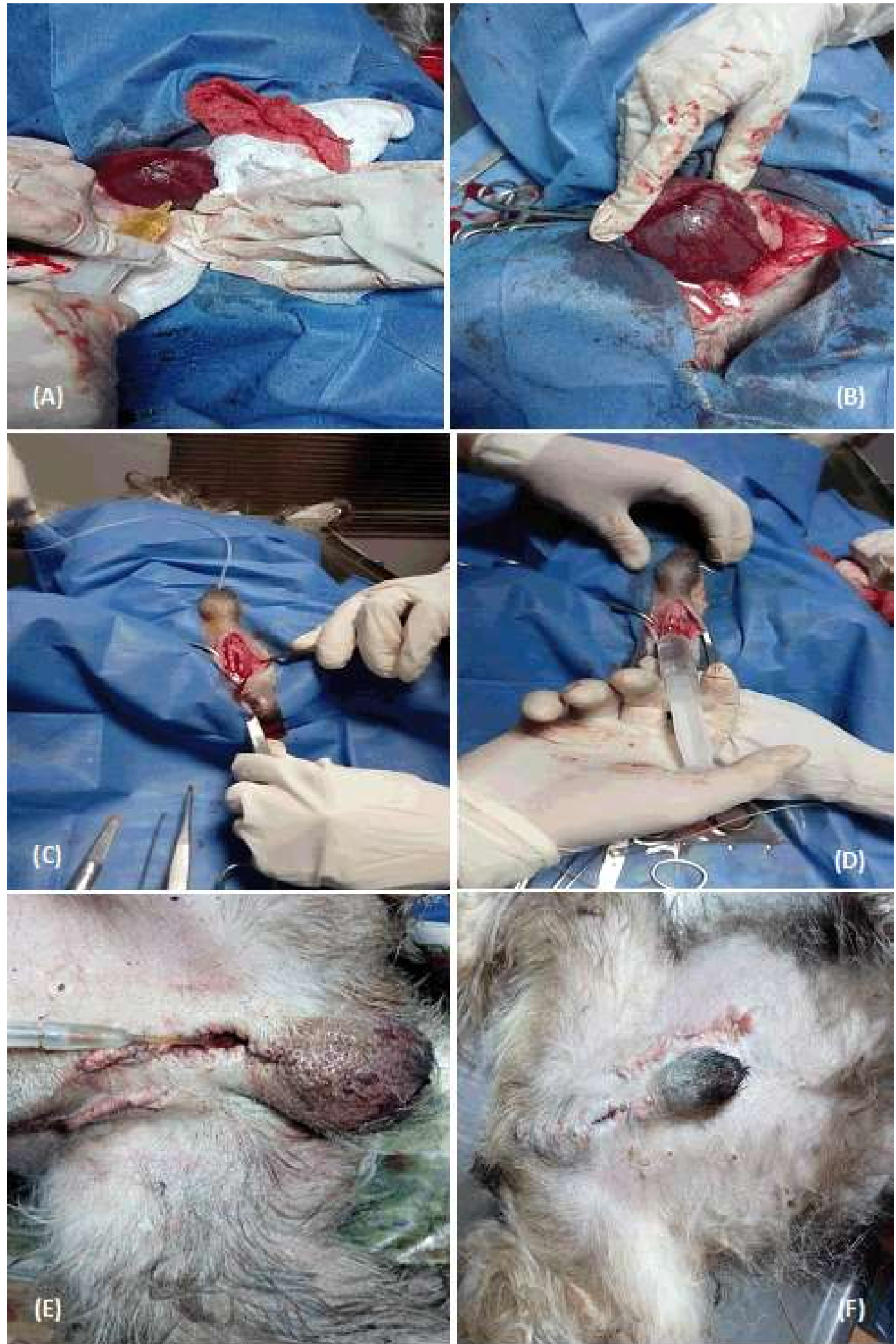

Figura 2. Procedimiento quirúrgico en perro Schnauzer de 7 años con uropatía obstructiva. (A) Cistocentesis in situ; (B) Exposición de la vejiga urinaria; (C) Uretrotomía y sonda uretral; (D) Uretrotomía y lavado uretral; (E) Uretrostomía y colocación de sonda uretral; (F) Uretrostomía, 10 días después de la cirugía 
en el perro y el gato. Parte 1: evaluación del paciente con ERC. Clin Vet PeqAnim 32:215-223.

5. Diaz-Espineira MM, Viehoff $F W$, Nickel RF. 1998. Idiopathic detrusor urethral dyssynergia in dogs: A retrospective analysis of 22 cases. J Small Anim Pract 39: 264-270. doi: 10.1111/j.1748-5827.1998.tb03648.x

6. Hostutler RA, Chew DJ, Eaton KA, DiBartola SP. 2004. Cystoscopic appearance of proliferative urethritis in 2 dogs before and after treatment. J Vet Intern Med 18: 113-116. doi: 10.1892/ 0891-6640(2004)18<113:caopui>2.0.co;2

7. Inkelmann MA, Kommers GD, Trost ME, Barros CS, Fighera RA, Irigoyen LF, Silveira IP. 2012. Lesions of the urinary systems in 1.063 dogs. Pesq Vet
Bras 32: 761-771. doi: 10.1590/S0100736X2012000800015

8. Milgram J. 2017. Urethrostomy in male dogs. In: Complications in small animal surgery. John Wiley. p 497-499.

9. Moroff SD, Brown BA, Matthiesen DT, Scott RC. 1991. Infiltrative urethral disease in female dogs: 41 cases (19801987). JAm Vet Med Assoc 199: 247-251.

10. Pedrozo R, Quintana GI, Bazán A, Florentín M. 2010. Valores hematológicos de referencia en caninos adultos aparentemente sanos, que concurren a una clínica privada de Asunción. Mem Inst Investig Cienc Salud 8: 5-13.

11. Smeak DD. 2000. Urethrotomy and urethrostomy in the dog. Clin Tech Small Anim Pract 15: 25-34. doi: 10.1053/ svms.2000.7301 